\title{
Healing of Polymeric Solids by Supramolecular Means
}

\author{
Laura N. Neumann ${ }^{\star}$, Christoph Weder, and Stephen Schrettl ${ }^{\star}$ \\ §SCS-Metrohm Award for best oral presentation in Polymers, Colloids \& Interfaces
}

\begin{abstract}
Equipping a polymeric material with the ability to heal an inflicted damage is a crucial advantage for many applications. The incorporation of reversible and dynamic supramolecular interactions into polymeric systems has proven to be a promising route towards such materials. In this article, recent developments in the field of healable materials are highlighted with a particular focus on the design principles, driving forces, and mechanisms that allow healing to occur.
\end{abstract}

Keywords: Healing $\cdot$ Healable materials $\cdot$ Non-covalent interactions $\cdot$ Supramolecular polymers

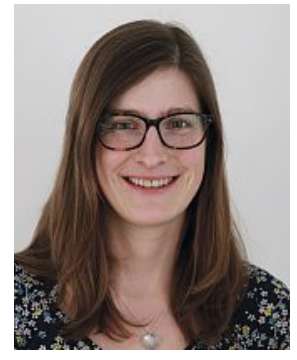

Laura Neumann studied at the Eindhoven University of Technology (the Netherlands) where she graduated in 2015 with a focus on supramolecular chemistry. Currently, she is a PhD student in Prof. Christoph Weder's research group at the Adolphe Merkle Institute of the University of Fribourg (Switzerland) within the framework of the National Center of Competence in Research Bio-Inspired Materials. Her interests include the synthesis and characterization of supramolecular polymers with a special focus on stimuli-responsive metallosupramolecular polymers.

\section{Introduction}

Continuous exposure to stresses inevitably causes materials to wear and experience damage at some point during use. Healable polymer materials that allow for the repair of defects have accordingly attracted considerable interest, ${ }^{[1-9]}$ and potential applications of such materials range from engineering plastics to soft electronics and biomedical technology. ${ }^{[10]}$ In recent years, significant progress has been made in the preparation of such polymers and different principles and processes that impart a material with the ability to heal have been established. Generally, two categories can be distinguished based on the type of healing mechanism. Materials that display extrinsic healing are those that feature (encapsulated) reactive components that become activated when damage occurs. ${ }^{[11]}$ By contrast, intrinsic healing is observed for materials that contain reversible covalent bonds or dynamic noncovalent interactions whose reconfigurations (partially) restore the structure after damage. ${ }^{[12]}$

In the context of this article, we focus on polymeric solids that display intrinsic healing on the basis of non-covalent (supramolecular) interactions and, following the definition by Rubinstein and coworkers, "can revert to their original state with full or partial recovery of mechanical strength".[13] The reversible nature of non-covalent interactions potentially allows these polymers to undergo many repair cycles without diminished properties, ${ }^{[14]}$ rendering them particularly interesting candidates for the development of healable materials. Furthermore, a broad variety of in- teractions can be exploited, including metal-ligand complexation, hydrogen-bonding, ionic-, $\pi$-, hydrophobic-, and host-guest interactions, which feature variable strengths that enable the development of materials with tailored properties.

Notably, a conceptual division has been made between materials that display stimuli-induced (e.g. thermal, optical, or $\mathrm{pH}$ ) or autonomous healing, even though both are often referred to as 'self-healing' materials. ${ }^{[15]}$ As discussed below, the conditions for efficient healing are fundamentally a question of relative activation energies, and 'self-healing' will be herein exclusively used for materials that heal at ambient conditions without an external stimulus. As the number of self-healing or healable supramolecular polymers has virtually exploded in the last decade, we illustrate the state of the field of solid healable supramolecular polymers by highlighting selected recent examples. The healing process is then outlined and discussed based on practical findings on different length scales, i.e. from the macroscopic to the microscopic level. Since the healing of gels is significantly facilitated by large quantities of solvents, the latter are outside the scope of the present article and the interested reader is referred to a recent review on this class of materials. ${ }^{[16]}$

\section{Supramolecular Interactions for Healing}

As outlined above, a broad range of supramolecular motifs have proven useful in the development of healable polymer materials. ${ }^{[1,2]}$ In metallosupramolecular materials, metal ions are coordinated to suitable ligands that are covalently incorporated into a polymer backbone. A variation of the metal-ion or ligand gives rise to different coordination geometries and allows for tuning of the binding strength of such complexes across a large range. ${ }^{[17,18]}$ For example, healing efficiencies of poly(ethylene-co-butylene) with 2,6-bis(1'-methylbenzimidazolyl)pyridine (Mebip) ligands at the termini upon exposure to UV light were dependent on the choice of metal ion, ${ }^{[19]}$ with more dynamic 3:1 Mebip:La ${ }^{3+}$ complexes displaying improved healing over 2:1 Mebip: $\mathrm{Zn}^{2+}$ complexes. In both cases, the soft polymer core phase separates from the hard phase of the metal-ligand complexes, which reinforces the materials and impedes creep under ambient conditions (see Section 3.3). In a recent effort that exploited the multi-dentate nature of metal-ligand complexes, Bao and coworkers employed 
poly(dimethylsiloxane)s that were cross-linked by coordination complexes between 2,6-pyridinedicarboxamide ligands and iron(III) ions (Fig. 1a). ${ }^{[20]}$ The complexes feature interactions of different strengths, i.e. a strong pyridyl-iron coordination as well as weaker interactions between the nitrogen and oxygen atoms of the carboxamide groups and the iron ion. Consequently, the ironcarboxamide bond interactions can break and reform, while the iron centers remain attached to the pyridine ligands. This enables retaining a high elasticity in the cross-linked material through a dynamic and reversible unfolding and refolding of chains, while at the same time allowing for self-healing to occur. A healing efficiency of $90 \pm 3 \%$ was observed after 48 hours at ambient conditions.

Individual hydrogen bonds are rather weak when compared to metal-ligand complexes and usually insufficient for the formation of supramolecular polymers. Consequently, moieties that combine multiple hydrogen bonds in a directional manner such as the strongly dimerizing quadrupole hydrogen bonding 2-ureido-4[1H]-pyrimidinone (UPy) and sextuple hydrogen bonding Hamilton wedge-barbiturate pair are employed. ${ }^{[1,2,5,21]}$ Nonetheless, the vast majority of healable materials based on hydrogen-bonding interactions are thermoplastic elastomers, with relatively low strength and stiffness, also because the binding motifs are usually combined with low-glass-transition-temperature telechelics. ${ }^{[9]}$ With the goal to access more rigid healable polymers, the preparation of healable supramolecular polymer glasses was recently reported. ${ }^{[22]}$ Thus, functionalizing a low-molecularweight monomer with three UPy moieties furnished materials with a room temperature storage modulus of up to $3.65 \pm 0.51 \mathrm{GPa}$ that heal upon exposure to UV irradiation. Further expanding the regime of attainable properties, Aida and coworkers described a novel class of healable poly(ether-thiourea)-based materials with high mechanical robustness. [23] The high density of hydrogen bonds in these low-molecular weight poly(ether-thioureas) re- sulted in a highly cross-linked, yet healable material. The particular zigzag hydrogen-bonding arrays between thiourea moieties prevented their crystallization into hard domains that arrests the dynamic characteristics of the non-covalent interactions in related materials (Fig. 1b). Among the derivatives investigated, polymers that feature a triethylene glycol spacer between neighboring thiourea motifs were found to display a particularly interesting combination of properties. The spacer was found to facilitate the exchange between hydrogen-bonded pairs, resulting in materials that completely heal under ambient conditions within 6 hours, while at the same time showing a high mechanical robustness of up to $45 \pm 8 \mathrm{MPa}$ and elongation at break of up to $393 \pm 5 \%$.

The incorporation of multiple orthogonal types of supramolecular interactions in a single material is an approach towards materials that exploit the dynamics between different types of non-covalent interactions for efficient healing. ${ }^{[2]}$ Moreover, this promises to enable both a fine-tuning of materials properties and the development of more complex and hierarchical networks. For example, hydrogen-bonding interactions and metal-ligand complexes were incorporated into the same material by functionalization of the termini of a three-armed poly(propylene oxide) with either Mebip ligands or UPy groups. [24] The cross-linking was established by complex formation between Mebip ligands and $\mathrm{Zn}^{2+}$ ions, while the UPy dimerization furnishes a separate, hydrogen bonding network (Fig. 1c). The independent disassembly of metal-ligand complexes and UPy dimers allowed for selective and tunable healing behavior in addition to double and triple shape-memory effects.

The highlighted examples showcase that reversible and dynamic non-covalent interactions are exceptionally suitable for the development of healable polymer materials. The range of available interaction types with variable binding characteristics allows systematically tuning the conditions of healing and the materials properties. Recent efforts have significantly expanded the attain- a)
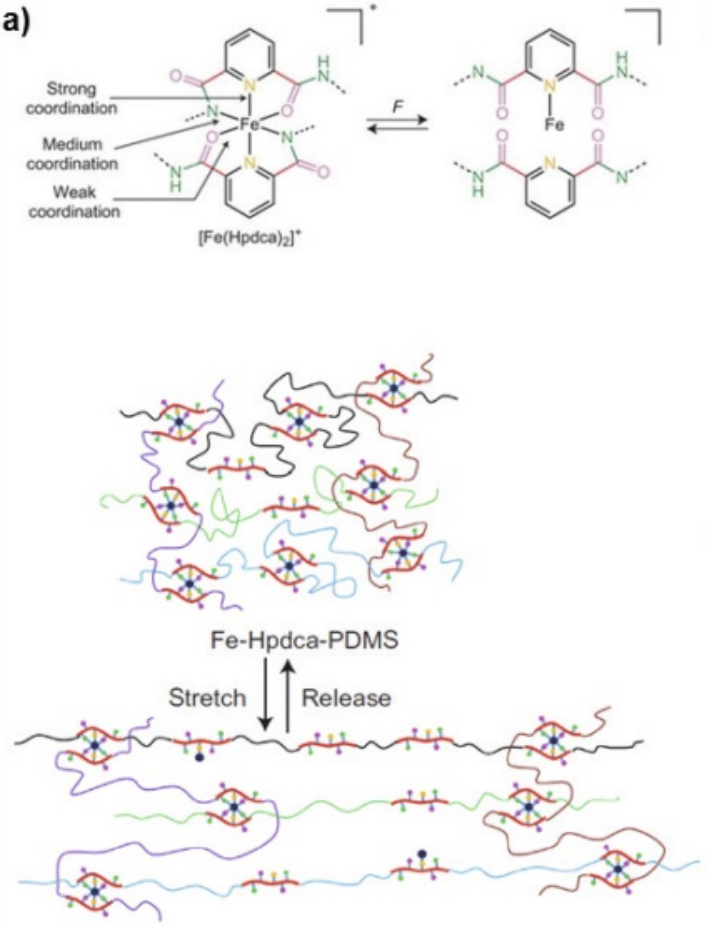

b)

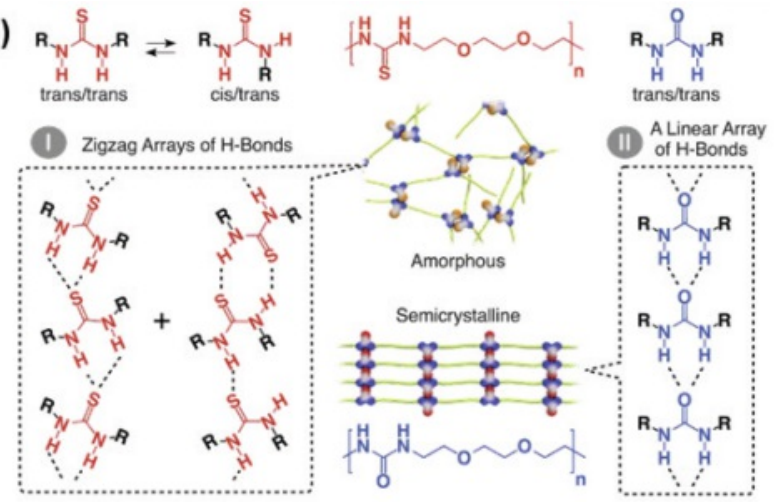

c)

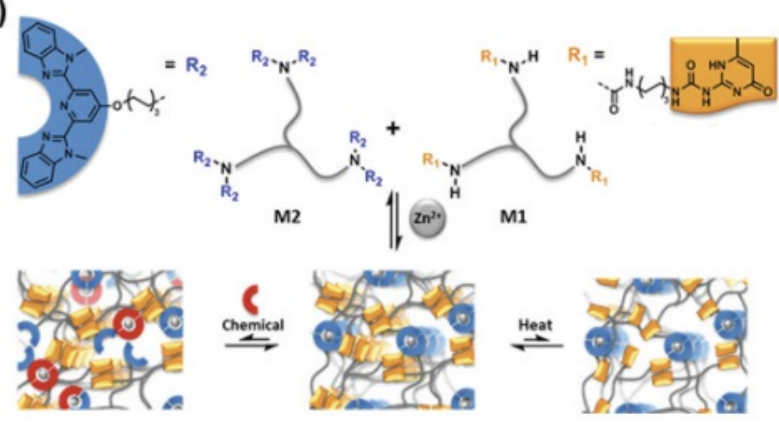

Fig. 1. (a) Schematic structure of the 2,6-pyridinedicarboxamide/iron-complexes ( $\left.\mathrm{Fe}(\mathrm{Hpdca})_{2}{ }^{+}\right)$undergoing reversible rupture and reformation during tensile deformation (top) and the structure of the material and proposed mechanism for chain folding and sliding (bottom). Reproduced and adapted with permission from ref. [20]; Copyright 2016 Nature Publishing Group. (b) Schematic representations of the hydrogen bonding interactions in polymers that feature either thiourea or urea moieties. Reproduced and adapted with permission from ref. [23]; Copyright 2018 American Association for the Advancement of Science. (c) Schematic structure of the three-armed poly(propylene oxide) with either Mebip ligands or UPy groups and the network formation by supramolecular cross-linking (Mebip/ $\mathrm{Zn}^{2+}$ complexes or UPy dimerization). Cartoons of the orthogonal responses of such networks to chemical stimuli or heat. Reproduced and adapted with permission from ref. [24]; Copyright 2018 American Chemical Society. 
able property regime of this class of polymers, and the healing process and key aspects that impart materials with the ability to heal will be discussed in the following.

\section{Elucidating the Healing Process}

In order to optimize the properties of healable materials, an understanding of the healing process is required. Essentially, healing of supramolecular materials takes place via dissociation and recombination of the supramolecular motifs, and the process is accordingly influenced by the strength and dynamic character of the employed interactions. In solid films, however, the motions of the polymer chains, as well as additional secondary factors such as phase separation and crystallization play an important role and complicate the analysis of healing processes. In this section, first the theoretical framework for healing will be discussed, followed by an analysis of the processes during healing at different length scales starting at the macroscopic level, and then zooming in towards the microscopic level.

\subsection{Theoretical Considerations}

A thermodynamic description of the conditions necessary for healing of supramolecular materials was provided by Yang and Urban. ${ }^{[3]}$ In their approach, the change in free energy $\Delta G$ (assuming $\Delta G=-T \Delta S$ ) was correlated with the concentration of free (surface A) and bound chain ends (surface B), the number of chain segments $N$, as well as the flexibility $f$ of chains as depicted in Fig. 2a. At sites where damage occurred, chains of lower molecular weight with a high mobility (above the grey $\Delta G=0$ plane) are present in high concentrations. This leads to spontaneous flow of chains and repair of the damage. As the healing progresses and non-covalent motifs recombine, an increase of the number of chain segments $N$ and a concomitant decrease of the individual chain flexibility $f$ is observed. The process continues up to a threshold value of a given $N$ when a stable equilibrium state $(\Delta G=0)$ is reached.

On the basis of models that are employed to describe macromolecular motion in polymeric solids, healing is considered to essentially rely on the diffusion of polymer chains across an interface. ${ }^{[3]}$ De Gennes derived the molecular weight dependence of the diffusional motion of entangled macromolecules. The time required for complete renewal of the chain conformation was to be $t_{\mathrm{r}} \propto M^{3}$ on the basis of the reptation model, according to which the movement of individual polymer chains is constrained to a 'tube' with sideways movements restricted by the tube diameter.[25] Chain diffusion across an interface is the main factor that drives the healing process, and the healing time therefore correlates with $t$ that scales exponentially with the molecular weight. Considering the processes that occur throughout healing a polymer-polymer interface, Wool and O'Connor described five different phases: segmental surface rearrangements, surface approach, wetting, diffusion, and randomization. ${ }^{[26]}$ The intrinsic equilibrium of supramolecular polymers between chain-extended species and macromonomers of lower molecular weight is the driving force for healing processes in these materials. The challenge of a theoretical description of healing processes in supramolecular systems is to account for the influence of the dissociation and recombination events of the supramolecular binding motifs, which change the apparent molecular weight and thereby significantly influence diffusive chain motion.

A model developed by Leibler, Rubinstein, and coworkers considers classical telechelic polymers with binding motifs at the termini for which one end ('sticker') of each chain is fixed in space, whereas the other end is available to form a pairwise reversible bond. ${ }^{[13]}$ As illustrated in Fig. 2b, damaging the material breaks supramolecular bonds and consequently increases the concentration of free binding motifs at the freshly ruptured surface. Healing then takes place by polymer diffusion and recombination of these motifs. The diffusive motion of supramolecular polymers can be described as segmental chain dynamics (Rouse dynamics), and a distinction is made between non-covalent interactions of different bond strengths $\varepsilon$. Thus, systems with weak $\left(\varepsilon<<k_{\mathrm{b}} T \ln N\right)$ or intermediate $\left(k_{\mathrm{b}} T \ln N<\varepsilon<2 k_{\mathrm{b}} T \ln N\right)$ bond strengths always feature a significant fraction of dissociated binding motifs in the bulk, while materials with high bond strengths $\left(k_{\mathrm{b}} T \ln N<<\varepsilon\right)$ only have very few dissociated binding motifs. In the case of weak or intermediate bond strengths, a high availability of binding sites renders the recombination independent of their concentration and dominated by the chain dynamics (anomalous subdiffusive Rouse dynamics). In the case of high bond strengths, however, recombination is mainly limited by the low availability of free a)

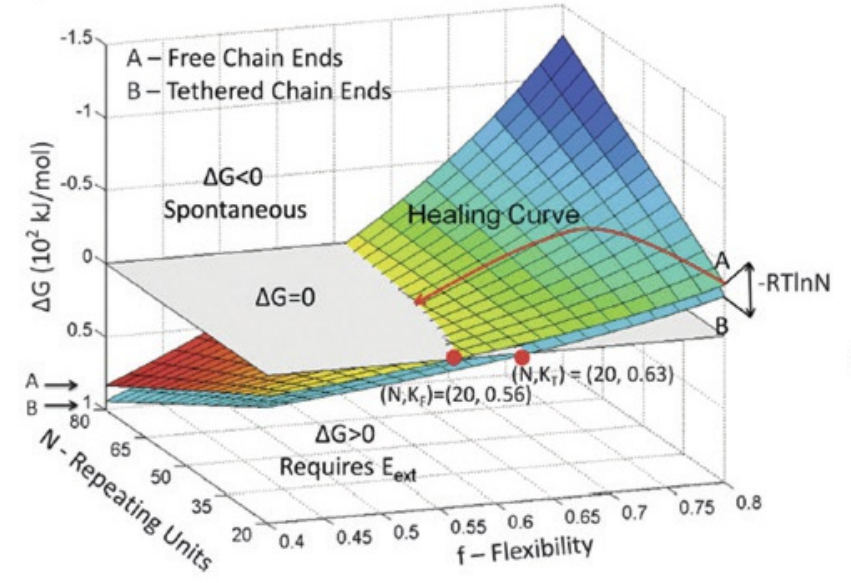

b)

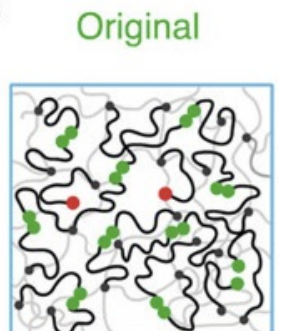

c)

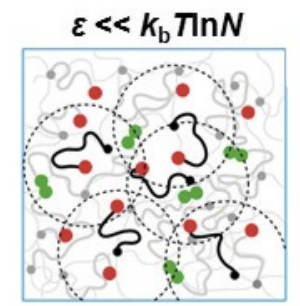

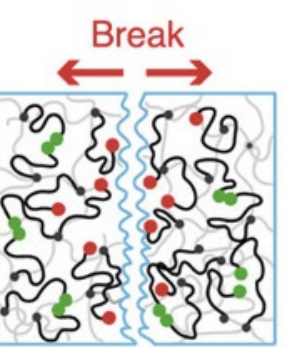

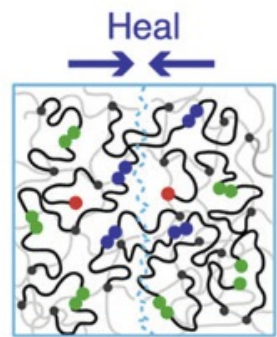

$k_{\mathrm{b}} \pi \mathrm{n} N \ll \varepsilon$

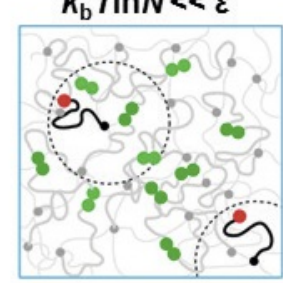

Fig. 2. (a) Plot of the changes in Gibbs free energy $(\Delta G=-T \Delta S$ ) for free (surface $A$ ) and tethered chain ends (surface $B$ ) as a function of the number of polymer chain segments $(N)$ and their flexibility parameter $f$. The grey plane represents $\Delta G=0$. Reproduced with permission from ref. [3]; Copyright 2013 The Royal Society of Chemistry. (b) Schematic representation of the healing process of a supramolecular polymer. Damaging the material results in unbound moieties at the ruptured surface (red circles) whereas these moieties re-associate once they are brought into contact, resulting in healing of the material (blue pairs of circles). Reproduced with permission from ref. [13]; Copyright 2013 The American Chemical Society. (c) Schematic representation of polymers with weak bond strength (left) and high bond strength (right). In the first case, there are many unbound moieties (red circles), whereas in the latter, there are almost none. Reproduced with permission from ref. [13]; Copyright 2013 The American Chemical Society. 
binding sites. Thus, a 'hopping'-type mechanism that requires an exchange of binding partners is employed to describe the recombination of two strong binding motifs (Fig. 2c). Accordingly, healing in supramolecular polymers is assumed to be based on the high energy of damaged interfaces and is an efficient process under conditions where the binding motifs are sufficiently dynamic to provide for a high concentration of available binding sites or a rapid exchange between binding partners.

\subsection{The Role of Interfacial Properties}

On a macroscopic level, the initial adhesion between damaged surfaces is an important step in the healing process. However, adhesion alone does not result in efficient healing with restoration of bulk mechanical properties since a significant fraction of binding motifs may recombine on the same interface, instead of recombining with motifs on the opposite interface. Thus, the number of free binding motifs at a damaged surface is expected to decrease with waiting time after fracture and low concentrations are present at unfractured and equilibrated surfaces. ${ }^{[13]}$ Indeed, various supramolecular systems display a decreased healing efficiency with increased waiting time after damage. ${ }^{[27,28]}$ For example, the healing efficiency in glassy materials with reversible hydrogen bonding interactions between complementary amidoethyl imidazolidone, di(amido ethyl) urea, or diamido tetraethyl triurea functionalized fatty acid dimers was observed to significantly decrease when waiting for 6 or 18 hours before fractured pieces were brought into contact.[28]

Considering that the interface of freshly damaged samples is assumed to have a high surface energy, a correlation between adhesive strength and surface energy was investigated for healable polymers. Thus, a self-healing poly(hydroxyethyl acrylate) and poly(ethylene glycol methacrylate) with $10 \mathrm{~mol} \%$ of hydrogenbonding UPy motifs and a variable side chain length showed high surface energies, but no direct relation between surface energy and adhesive strength was found. ${ }^{[29]}$ On the contrary, an increase of both the surface energy and improved adhesive properties was reported upon increasing the number of hydrogen bonds in a side functionalized poly(butyl acrylate) with 4.0 and $7.2 \mathrm{~mol} \%$ of UPy side chains. ${ }^{[30]}$ While a clear relation between surface energy and adhesive properties could not be established for these systems, efficient healing independent of the waiting time was reported for the poly(dimethylsiloxane) with 2,6-pyridinedicarboxamide- $\mathrm{Fe}^{3+}$ metal-ligand coordination complexes. ${ }^{[20]}$ Thus, after a waiting time of 24 hours and subsequent healing for 48 hours at room temperature, the healing efficiency was found to be $90 \pm 3 \%$, a value similar to the healing efficiency of freshly damaged samples. Moreover, even undamaged surfaces of this material were found to heal with an efficiency of up to $57 \pm 2 \%$, suggesting that a dynamic exchange of bonds is in this case taking place even at equilibrated surfaces. One might conclude that the initial adhesion between the surfaces is an important factor, but healing efficiency and recovery of materials properties appears to more heavily depend on the dynamic nature of the employed interactions.

\subsection{Structural Considerations}

In addition to the non-covalent cross-linking itself, the incorporation of supramolecular binding motifs into soft polymer matrices often furnishes secondary interactions as the moieties aggregate, phase separate, and possibly crystallize. Effectively, this microphase separation into a hard phase of binding motifs acts as an additional physical cross-linking of the typically soft polymer phase. ${ }^{[31]}$ The latter is generally advantageous for the mechanical properties of supramolecular polymers and can prevent the unwanted creep often observed in materials composed of a soft polymer core and intrinsically dynamic supramolecular interactions. ${ }^{[27]}$ However, 'freezing' of non-covalent interactions into a hard phase dramatically alters their dynamics and consequently the healing properties. Healing in phase-separated materials can in principle take place either via dissociation and recombination of individual supramolecular interactions or by disordering and reformation of the hard phase, i.e. independent of the reversible non-covalent interactions. ${ }^{[32]}$ In order to gain insights into the relation between these different relaxation processes and the healing kinetics, investigations were performed with a telechelic poly(isobutylene) carrying hydrogen-bonding barbituric acid groups at the termini. ${ }^{[33]}$ Thus, bulk samples of this polymer feature micellar aggregates of the binding motifs, and the interconnected nature of these aggregates was found to increase the mechanical strength of the material that displays self-healing properties. Detailed investigations by nonlinear rheology showed that the time-scale of macroscopic healing thereby correlates with the kinetics of the exchange of binding motifs between the micellar aggregates. Similarly, Rowan and coworkers investigated a telechelic poly(tetrahydrofuran) that had been terminally functionalized with adenine or cytosine hydrogen-bonding moieties and it was shown that the binding motifs phase segregate from the soft phased formed by the telechelic polymer. ${ }^{[34]}$ The relaxation processes of this material were found to strongly depend on the dissociation frequency of the binding motifs from the hard phase, rather than the dynamics of the non-covalent interactions.

In phase-separated block copolymers, the healing properties can be influenced by deliberately placing reversible supramolecular interactions in either the hard or the soft phase. Thus, Binder and coworkers investigated a multiblock copolymer with soft and hard segments that featured barbiturate/ $\alpha, \omega$-Hamilton wedge interactions at the phase boundary. ${ }^{[35]}$ In this system, samples were heated to temperatures close to the $T_{\mathrm{g}}$ of the hard domains in order to efficiently heal the material. On the other hand, Guan and coworkers incorporated zinc-imidazole interactions in the soft segments of a hard/soft two-phase brush copolymer and observed efficient self-healing. ${ }^{[36]}$ The latter was attributed to the combination of the fast ligand exchange kinetics of these types of metalligand complexes and to the mobility of the soft polymer segments at ambient conditions. Thus, the dynamic binding characteristics of the non-covalent interactions and polymer diffusion dominate the healing efficiency when the binding motifs are sufficiently mobile. If the latter themselves phase separate into a hard domain, mobility is restricted by the dissociation kinetics from the hard phase as the limiting factor.

\subsection{The Role of the Binding Strength}

Typically, the binding strength of supramolecular interactions is characterized by their equilibrium association constant $K_{\mathrm{a}}$ in solution. ${ }^{[37]}$ However, the solution-phase association between binding motifs is not necessarily a reliable measure for the behavior in the solid state. As outlined in the preceding section, phase separation can play a crucial role in the solid state, but additional effects might be at play as illustrated by a comparison of the association strength between telechelic poly ( $n$-butyl acrylate)s functionalized at the termini with either thymine (THY) or 2,6-diaminotriazine (DAT) as hydrogen bonding motifs. ${ }^{[38]}$ While self- and hetero-complementary binding can occur between these motifs, the hetero-complementary THY-DAT hydrogen bonds are found to preferentially form in solution. In the polymer melt, this preference was found to be negligible. Thus, self- as well as hetero-complementary binding was observed and resulted in similar bulk material properties. The variation in solution phase binding strengths appears to be compensated by secondary effects in the polymer melt, possibly through differences in polarity, aggregate formation, or related secondary interactions between binding motifs.

In an effort to determine the influence of the supramolecular binding strength on the dynamics of polymer networks, Seiffert and coworkers studied poly( $N$-isopropylacrylamide)s functional- 
ized with supramolecular moieties of varying strengths. ${ }^{[39]}$ The polymers were non-covalently cross-linked by using either motifs based on the relatively weak bis-maleimide/diaminotriazine hydrogen bonding interactions, medium strength cyanuric acid/ Hamilton wedge hydrogen bonding interactions, or strong metalligand complexes between terpyridine ligands and $\mathrm{Mn}^{2+}$ ions. Investigations of gels based on these polymers that featured fluorescence labeled chains allowed for a comparison of the chain diffusion coefficients. Their findings showed that the diffusive properties were only marginally influenced by the strength of the noncovalent bonds and that the overall dynamics of the systems were instead mainly controlled by the intrinsic polymer chain dynamics. Thurn-Albrecht, Binder, and coworkers similarly observed a significant influence of the polymer behavior on the properties of non-covalent interactions while investigating different polymers that were connected into supramolecular diblock copolymers by Hamilton wedge-type hydrogen bonds at one end of each polymer chain. ${ }^{[40]}$ Thus, the hydrogen bonding motifs were placed at the phase boundary between the polymers and an enhanced dissociation of the non-covalent bonds was observed for those polymer combinations that display an increased tendency for phase segregation.

The thermodynamic strength of the binding of non-covalent interactions is significantly influenced by the characteristics of the polymer backbone. For the process of healing, however, dissociation and re-association kinetics are much more important than the thermodynamic strength of the equilibrium structures. This was strikingly demonstrated by Craig and coworkers who compared the properties of poly(4-vinylpyridine) that had been cross-linked with pincer-type complexes of $\mathrm{Pd}^{2+}$ or $\mathrm{Pt}^{2+}$ ions with two different ligands of varying steric hindrance. ${ }^{[41,42]}$ Investigations in solution showed that the rate of ligand exchange was two orders of magnitude lower with sterically hindered ligands, while the corresponding association constants of the complexes remained almost unchanged. A characterization of gels of the materials showed that the altered dynamics of the non-covalent cross-links of comparable strength had a pronounced influence on the viscoelastic properties of the supramolecular networks. Interestingly, further studies with these systems showed that the bulk mechanical properties are more significantly influenced by the frequency of crosslink dissociations and, hence, relaxations of the network structure, while the fraction of dissociated binding motifs was less consequential.[43] These findings nicely confirm the theoretical models in that an enhanced ability of chain segments to diffuse is the key parameter that determines a material's healing efficiency.

\section{Conclusions}

As hopefully illustrated by the examples highlighted in this article, the reversible and dynamic characteristics of non-covalent interactions are exceptionally suited for the development of polymer materials that display autonomous or stimuli-induced healing with often complete recovery of the bulk mechanical properties. Based on the available range of interactions with their variable binding characteristics, the material properties and the conditions under which healing occurs can be readily tuned. Fundamental investigations of healable polymer systems offer some guidelines with respect to the relation between the characteristics of the reversible non-covalent interactions, the healing process, and the key aspects that impart materials with the ability to heal. Confirming theoretical considerations, the ability of the polymer chains to diffuse appears to play the most important role for an efficient healing. In order to rationally design healable materials, it is therefore important to consider the type of employed polymer and combine the latter with non-covalent binding motifs that are suitable for a dynamic reversible cleavage under the desired conditions of healing. In such tailored polymer networks the reversible cleavage should then lead to a high mobility of the cor- responding polymer backbone and thereby result in very efficient healing. ${ }^{[4]}$ While the continued interest in healable polymers has led to the development of many new materials, only few examples so far meet the challenge of offering the ability to readily heal in combination with desirable mechanical properties, such as those attainable with high molecular weight polymers or covalently cross-linked polymer networks. As outlined in the present article, the current level of understanding of healing processes offers useful guidelines for the development of materials that combine desirable materials properties with the ability to efficiently heal macroscopic damage. In this context, a wide range of technological applications is expected to benefit from polymeric materials that allow to improve the lifetime of components, their recycling, as well as reprocessing.

\section{Acknowledgements}

The authors would like to thank Dr. Ilja Gunkel for the helpful discussion. We also gratefully acknowledge financial support through the National Center of Competence in Research Bio-Inspired Materials, a research instrument of the Swiss National Science Foundation, the Adolphe Merkle Foundation, and from the European Research Council under the European Union's Seventh Framework Programme (FP7/20072013)/ERC grant agreement no. AdG 291490-MERESPO.

Received: January 23, 2019

[1] Y. Yang, M. W. Urban, Adv. Mater. Interfaces 2018, 5, 1800384.

[2] A. Campanella, D. Döhler, W. H. Binder, Macromol. Rapid Commun. 2018 39, 1700739.

[3] Y. Yang, M. W. Urban, Chem. Soc. Rev. 2013, 42, 7446.

[4] Y. Yang, X. C. Ding, M. W. Urban, Prog. Polym. Sci. 2015, 49-50, 34.

[5] F. Herbst, D. Dohler, P. Michael, W. H. Binder, Macromol. Rapid Commun. 2013, 34, 203.

[6] D. Döhler, P. Michael, W. Binder, 'Principles of Self-Healing Polymers', in 'Self-Healing Polymers', Wiley-VCH Verlag GmbH \& Co. KGaA, 2013.

[7] L. R. Hart, J. L. Harries, B. W. Greenland, H. M. Colquhoun, W. Hayes, Polym. Chem. 2013, 4, 4860 .

[8] S. Burattini, B. W. Greenland, D. Chappell, H. M. Colquhoun, W. Hayes, Chem. Soc. Rev. 2010, 39, 1973.

[9] L. M. de Espinosa, G. L. Fiore, C. Weder, E. Johan Foster, Y. C. Simon, Prog. Polym. Sci. 2015, 49-50, 60.

[10] M.-H. Wei, B. Li, R. L. A. David, S. C. Jones, V. Sarohia, J. A. Schmitigal, J. A. Kornfield, Science 2015, 350, 72.

[11] S. R. White, N. R. Sottos, P. H. Geubelle, J. S. Moore, M. R. Kessler, S. R. Sriram, E. N. Brown, S. Viswanathan, Nature 2001, 409, 794.

[12] J. Dahlke, S. Zechel, M. D. Hager, U. S. Schubert, Adv. Mater. Interfaces 2018, 5, 1800051.

[13] E. B. Stukalin, L.-H. Cai, N. A. Kumar, L. Leibler, M. Rubinstein, Macromolecules 2013, 46, 7525.

[14] L. Montero de Espinosa, S. Balog, C. Weder, ACS Macro Letters 2014, 3, 540 .

[15] K. M. Herbert, S. Schrettl, S. J. Rowan, C. Weder, Macromolecules 2017, 50 , 8845 .

[16] S. Strandman, X. X. Zhu, Gels 2016, 2, 16.

[17] A. Winter, U. S. Schubert, Chem. Soc. Rev. 2016, 45, 5311.

[18] I. Manners, 'Synthetic Metal-Containing Polymers', WILEY-VCH, 2004.

[19] M. Burnworth, L. Tang, J. R. Kumpfer, A. J. Duncan, F. L. Beyer, G. L. Fiore, S. J. Rowan, C. Weder, Nature 2011, 472, 334.

[20] C. H. Li, C. Wang, C. Keplinger, J. L. Zuo, L. Jin, Y. Sun, P. Zheng, Y. Cao, F. Lissel, C. Linder, X. Z. You, Z. Bao, Nat. Chem. 2016, 8, 618.

[21] F. Herbst, W. H. Binder, 'Self-Healing Polymers via Supramolecular, Hydrogen-Bonded Networks', in 'Self-Healing Polymers', Wiley-VCH Verlag GmbH \& Co. KGaA, 2013.

[22] D. W. Balkenende, C. A. Monnier, G. L. Fiore, C. Weder, Nat. Comm. 2016 7, 10995.

[23] Y. Yanagisawa, Y. Nan, K. Okuro, T. Aida, Science 2018, 359, 72.

[24] J. Sautaux, L. Montero de Espinosa, S. Balog, C. Weder, Macromolecules 2018, 51, 5867.

[25] P. G. d. Gennes, J. Chem. Phys. 1971, 55, 572

[26] R. P. Wool, K. M. O'Connor, J. Appl. Phys. 1981, 52, 5953.

[27] Y. Chen, A. M. Kushner, G. A. Williams, Z. Guan, Nat. Chem. 2012, 4, 467

[28] P. Cordier, F. Tournilhac, C. Soulie-Ziakovic, L. Leibler, Nature 2008, 451, 977.

[29] D. Zhu, Q. Ye, X. Lu, Q. Lu, Polym. Chem. 2015, 6, 5086.

[30] A. Faghihnejad, K. E. Feldman, J. Yu, M. V. Tirrell, J. N. Israelachvili, C. J. Hawker, E. J. Kramer, H. Zeng, Adv. Funct. Mater. 2014, 24, 2322. 
[31] R. J. Wojtecki, A. Nelson, J. Polym. Sci., Part A: Polym. Chem. 2016, 54, 457.

[32] S. Bode, B. Sandmann, M. D. Hager, U. S. Schubert, 'Metal-ComplexBased Self-Healing Polymers', in 'Self-Healing Polymers', Wiley-VCH Verlag $\mathrm{GmbH} \& \mathrm{Co} . \mathrm{KGaA}, 2013$.

[33] T. Yan, K. Schroter, F. Herbst, W. H. Binder, T. Thurn-Albrecht, Sci. Rep. 2016, 6, 32356 .

[34] S. Sivakova, D. A. Bohnsack, M. E. Mackay, P. Suwanmala, S. J. Rowan, J. Am. Chem. Soc. 2005, 127, 18202.

[35] S. Chen, N. Mahmood, M. Beiner, W. H. Binder, Angew. Chem. 2015, 127 , 10326.

[36] D. Mozhdehi, S. Ayala, O. R. Cromwell, Z. Guan, J. Am. Chem. Soc. 2014, $136,16128$.
[37] S. Seiffert, J. Sprakel, Chem. Soc. Rev. 2012, 41, 909.

[38] F. Herbst, W. H. Binder, Polym. Chem. 2013, 4, 3602

[39] S. Hackelbusch, T. Rossow, P. van Assenbergh, S. Seiffert, Macromolecules 2013, 46, 6273.

[40] S. Chen, T. Yan, M. Fischer, A. Mordvinkin, K. Saalwächter, T. ThurnAlbrecht, W. H. Binder, Angew. Chem. Int. Ed. 2017, 56, 13016.

[41] W. C. Yount, H. Juwarker, S. L. Craig, J. Am. Chem. Soc. 2003, 125, 15302.

[42] W. C. Yount, D. M. Loveless, S. L. Craig, Angew. Chem. Int. Ed. 2005, 44, 2746.

[43] W. C. Yount, D. M. Loveless, S. L. Craig, J. Am. Chem. Soc. 2005, 127, 14488. 